\title{
Numerical Simulation-Based Optimization of Contact Stress Distribution and Lubrication Conditions in the Straight Worm Drive
}

\author{
Y. He, ${ }^{a}$ G. F. Li, ${ }^{a, b, 1}$ Y. P. Zhao, ${ }^{c}$ Y. Sun, ${ }^{a, b}$ and G. Z. Jiang ${ }^{a, b}$
}

a The Key Laboratory of Metallurgical Equipment and Control of Ministry of Education, Wuhan University of Science and Technology, Wuhan, China

b Hubei Key Laboratory of Mechanical Transmission and Manufacturing Engineering, Wuhan University of Science and Technology, Wuhan, China

c School of Machinery and Automation, Northeastern University, Shenyang, China

1 ligongfa@wust.edu.cn

Tooth contact area optimization/meshing of the straight worm pair is vital for improving its operation efficiency. Geometric parameters of a worm helicoid were derived via the gear meshing theory using the equations of the instantaneous contact line. The visualization function and powerful computing capability of MATLAB are used for the numerical analysis where the instantaneous contact line distribution is simulated for particular worm drive parameters, which yields the induced curvature and slip angle in the direction of the line. The above results are used for further optimization of the meshing performance of the straight worm gear and identification of its influencing factors. A case study of the particular cylindrical worm gear in compliance with the GB 10085-1988 Standard of China demonstrate that a reasonable selection of worm gear parameters can extend the contact line and the working range of the tooth surface of the worm gear and/or make the contact line distribution along the worm wheel more uniform, thus improving the local meshing performance.

Keywords: straight worm drive, meshing performance, instantaneous contact line, helix angle, MATLAB.

\section{Notation}

a $\quad-$ center distance of a worm pair, $\mathrm{mm}$

$r_{0} \quad-$ cylinder radius, $\mathrm{mm}$

$i_{12} \quad-$ transmission ratio of a worm drive

$\alpha \quad-$ helix angle, deg

$p \quad-$ helix parameter

$\vec{r}_{1} \quad-$ worm helicoid

$\theta, u \quad-$ parameters of worm helicoid

$\varphi_{1}, \varphi_{2}-$ rotation angles of worm pair, deg

$\Phi \quad-\quad$ meshing condition function

$\theta_{v t} \quad-$ slip angle, deg

$K_{12 N} \quad-$ instantaneous contact line direction induced curvature

$\psi \quad-$ curvature interference boundary function

$m \quad-$ modulus of worm

$K_{\alpha}, \tau_{\alpha}-$ curvature and short-range torsion of the worm helical plane in tangent direction

$\vec{v}_{12} \quad-$ relative speed of the worm pair at the meshing point

$\vec{N} \quad-$ normal vector of the instantaneous contact line 
Introduction. Worm drive reducers are compact, smooth, and provide relatively large transmission ratios. However, a high sliding velocity between helical teeth on the worm and worm gear meshing tooth surface results in severe tooth wear, large heat release, and pitting occurrence [1]. The correct meshing of worm gears is crucial for preserving an oil film: too small contact areas can result in the film rupture and consequent abrasive wear, whereas if the contact extends to the entry edge of the worm wheel, a lubricant can be scraped from the worm by the tooth edges.

Worm drives, including cylindrical or straight worms (Fig. 1a), cone or double enveloping worms (Fig. 1b), plane internal gear enveloping hourglass worm gear (Fig. 1c), and rolling conical teethed toroidal planet worm gears (Fig. 1d) have been deeply studied in theory and machining [2-4].



Single threaded

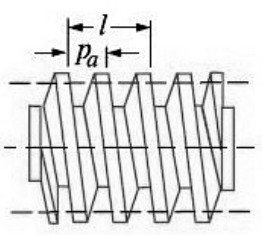

Double threaded

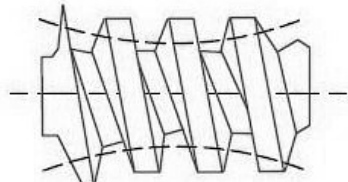

b

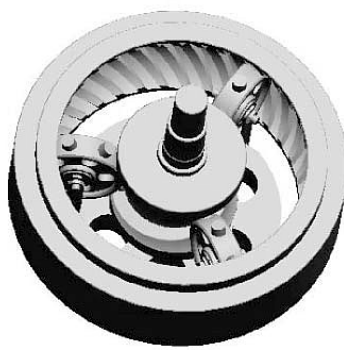

d

Fig. 1. Types of worm drives.

At present, with the rapid development of worm drives, the research on their structure reached saturation, and its focus was shifted to the error estimation, force analysis, and the parametric design of worm drives [5-7]. The analysis of worm drives is presently combined with the computer-aided design, wherein the software is used to simulate the driving and machining processes and the obtained virtual model of the worm gear is scrutinized from many aspects [8]. Thus, a numerical simulation of loaded line and point contacts in face gears performed by Li et al. [9] in 2005 has shown the superiority of a line contact over a point one, since it provides higher contact ratio, lower maximal load, lower loaded transmission error, and lower contact pressure. While the bearing capacity and driving efficiency of the worm gear mainly rely on the experimental results, the distribution of instantaneous contact line can be simulated, and the meshing performance of worm gear can be indirectly assessed via the numerical simulation $[9,10]$. On this basis, the influencing factors of meshing performance can also be analyzed, so that the mesh performance of worm gear can be improved by choosing the appropriate design parameters $[11,12]$. In this study, the numerical simulation of the instantaneous contact of the straight worm via MATLAB is performed, and the induced curvature and slip angle in the direction of the instantaneous contact line are calculated. Finally, the meshing performance of straight worm gear and its influencing factors are analyzed in detail. 
1. Worm Pair Parameters. Using the normal worm meshing theory [13], the correlation function can be expressed via parameters $u, \theta$, and $\varphi_{1}$. A set of worm parameters was selected as the reference object for analysis to specify the meshing quality of the worm gear [14]. In accordance to the GB 10085-1988 Standard of China on cylindrical worm gears, which applies to the cases where modulus $m$ is no less than $1 \mathrm{~mm}$, the major parameters were selected as shown in Table 1 [15].

$\mathrm{T}$ a b 1 e 1

Major Parameters of the Worm Pair under Study

\begin{tabular}{||c|c|c|c|c|c|c|c||}
\hline \hline Parameter & $a, \mathrm{~mm}$ & $z_{1}$ & $\alpha_{n}, \mathrm{deg}$ & $m, \mathrm{~mm}$ & $i_{12}$ & $x_{2}$ & $q$ \\
\hline Value & 100 & 1 & 20 & 5 & 31 & -0.5 & 10 \\
\hline Parameter & $p_{x}, \mathrm{~mm}$ & $p_{z}, \mathrm{~mm}$ & $d_{1}, \mathrm{~mm}$ & $d_{a 1}, \mathrm{~mm}$ & $d_{f 1}, \mathrm{~mm}$ & $\gamma, \mathrm{deg}$ & \\
\cline { 1 - 6 } Value & 15.708 & 15.708 & 50 & 60 & 38 & 5.71 & \\
\hline
\end{tabular}

In Table 1, the lead angle of the worm $\gamma$ is the helix angle $\alpha$, the helix parameters $p$, $u$ and cylindrical radius $r_{0}$ need to be calculated, these parameters are related to the helix angle $\alpha$, tooth profile angle $\alpha_{n}$, and root diameter of the worm $d_{f 1}$. The worm helicoid can be projected onto the plane $i O j$, as shown in Fig. 2, where the internal circle diameter is the worm root diameter and the external one is the worm addendum diameter $d_{a 1}$.

Assuming $z=0$, the projection of worm helicoid on the $i O j$ plane can be described as follows:

$$
\left\{\begin{array}{l}
x_{1}=-r_{0} \sin \theta+u \cos \alpha \cos \theta, \\
y_{1}=r_{0} \cos \theta+u \cos \alpha \sin \theta,
\end{array} \quad x_{1}^{2}+y_{1}^{2}=r_{0}^{2}+u^{2} \cos ^{2} \alpha .\right.
$$

When $u$ takes the minimum value, Eq. (1) can be reduced to

$$
x_{1}^{2}+y_{1}^{2}=r_{0}^{2}+u_{\min }^{2} \cos ^{2} \alpha=\left(r_{f 1}\right)^{2} \text {. }
$$

The relationship between $r_{0}$ and $u_{\text {min }}$ is depicted in Fig. 3, where $O A=r_{0}$, $C D=r_{f 1}=d_{f 1} / 2$, line $C D$ is parallel to the $x O y$ plane, $O B$ is the projection of $C D$ onto $x O y$, the angle between $A B$ and $A C$ lines is $\alpha$, the angle between $A C$ and $C D$ is $\alpha_{n}$, while the length of $A C$ is equal to $u_{\min }$.

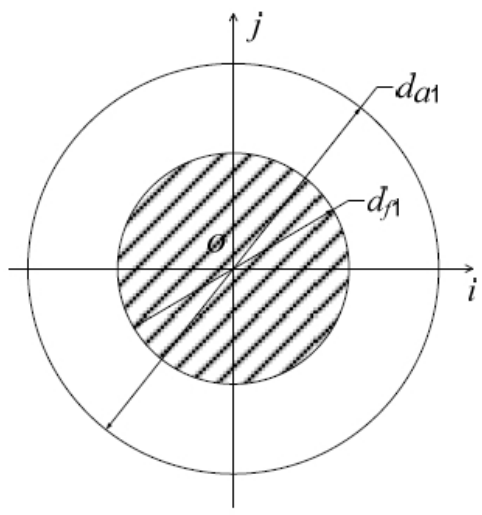

Fig. 2. Projection of the worm helicoid on the $i O j$ plane.

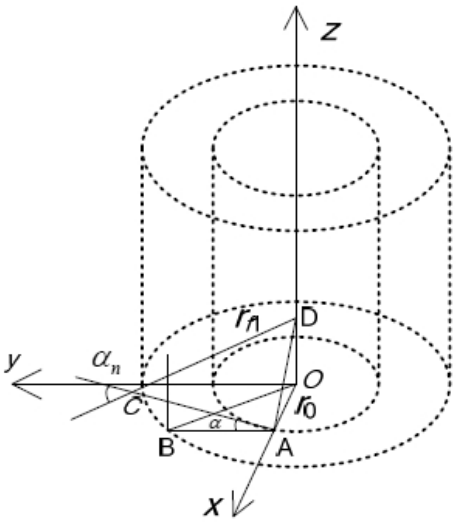

Fig. 3. Relationship between $r_{0}$ and $u_{\min }$. 
Substitution of the particular values of $r_{f 1}, \alpha_{n}$, and $\alpha$ from Table 1 into Eq. (2) yields the following value of $u_{\min }$ :

$$
u_{\text {min }}=\frac{r_{f 1} \cos \left(\alpha_{n}\right)}{\cos ^{2} \alpha}=\frac{19 \cos \left(20^{\circ}\right)}{\cos ^{2}\left(5.71^{\circ}\right)}=18.0327 \mathrm{~mm} .
$$

Then the value of $r_{0}$ can be obtained from Eq. (2) as follows:

$$
r_{0}=\sqrt{r_{f 1}^{2}-u_{\min }^{2} \cos ^{2} \alpha}=\sqrt{19^{2}-18.0327^{2} \times \cos ^{2}\left(5.71^{\circ}\right)}=6.2483 \mathrm{~mm} .
$$

Similarly, for the maximum value of $u$, we get

$$
u_{\max }=\sqrt{\frac{r_{a 1}^{2}-r_{0}^{2}}{\cos ^{2} \alpha}}=\sqrt{\frac{30^{2}-6.2483^{2}}{\cos ^{2}\left(5.71^{\circ}\right)}}=29.4884 \mathrm{~mm} .
$$

Next, the helix parameter $p$ can be obtained via the worm axial pitch, as follows:

$$
p=\frac{p_{x}}{2 \pi}=\frac{\pi m}{2 \pi}=\frac{m}{2}=2.5 \mathrm{~mm}
$$

By applying the derived parameters to the worm helicoid $\Sigma_{1}$, its shape can be calculated and plotted through the numerical simulation via the MATLAB drawing function, as is shown in Fig. 4a.

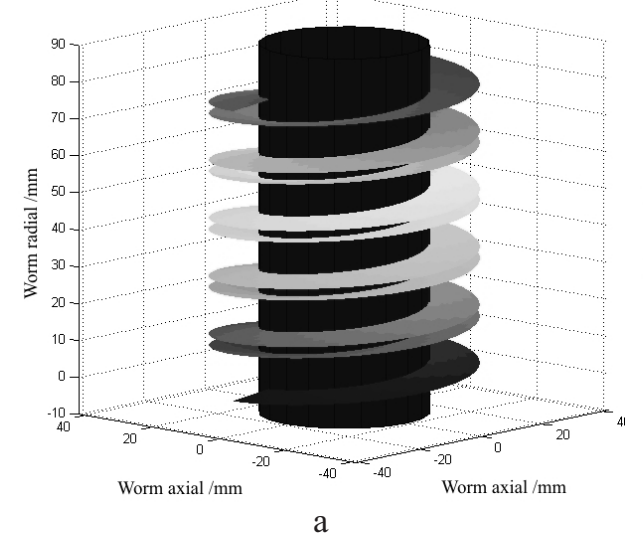

a

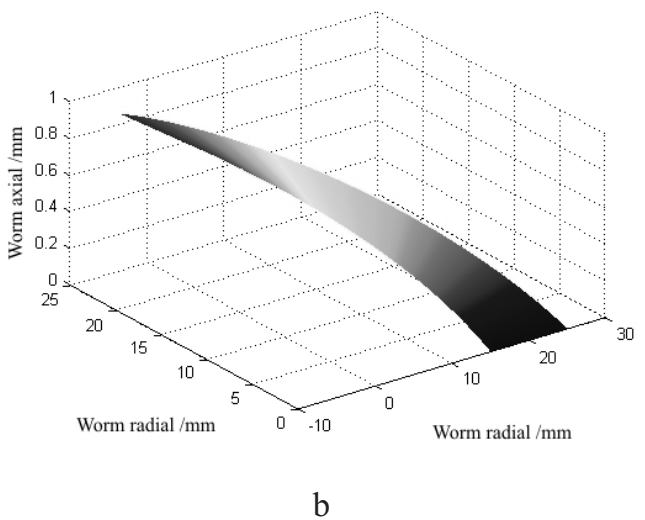

(a) and $\Sigma_{2}($ b)

According to the worm gear meshing condition, the worm gear meshing surface equation can be deduced as follows:

$$
\left\{\begin{array}{l}
x_{2}=r_{0} \cos \theta+u \cos \alpha \sin \theta+a \cos \varphi_{1}, \\
y_{2}=r_{0} \sin \theta-u \cos \alpha \sin \theta-a \sin \varphi_{1}, \\
z_{2}=p \theta-u \sin \alpha .
\end{array}\right.
$$

Applying the above parameters to the worm helicoid $\Sigma_{2}$, its shape can be derived through the numerical calculation and drawing function of MATLAB. Given the worm wheel surface repeatability, only one gear meshing surface shape is shown in Fig. 4b. 
2. Numerical Analysis of the Instantaneous Contact Line. The meshing limitation is the envelope line of the instantaneous contact line group for the worm helicoid $\Sigma_{1}$, as well as the dividing line between the meshing area and the invalid area in $\Sigma_{1}$. The meshing boundary equation can be written as

where

$$
\left\{\begin{array}{l}
\vec{r}_{1}=\vec{r}_{1}(u, \theta), \\
\Phi=0, \\
\Phi_{\varphi}=0,
\end{array}\right.
$$

$$
\begin{gathered}
\Phi=A_{1}(u, \theta) \cos \varphi_{1}-B_{1}(u, \theta) \sin \varphi_{1}+C_{1}(u, \theta)=0, \\
\Phi_{\varphi}=-A_{1}(u, \theta) \sin \varphi_{1}-B_{1}(u, \theta) \cos \varphi_{1}=0 .
\end{gathered}
$$

In MATLAB, we first define the parameter variables $r_{0}, u, \theta, \alpha, p, \varphi_{1}, a$, and $i_{12}$ and then derive $x_{1}, y_{1}, z_{1}, n_{x 1}, n_{y 1}, n_{z 1}$ and $A_{1}, B_{1}, C_{1}$ for these variables, to get the expressions of $\Phi$ and $\Phi_{\varphi}$ for these parameters and use the solver function in MATLAB to derive $u$ and $\theta$ in Eq. (4) via $\Phi$ and $\Phi_{\varphi}$.

After the values of $u$ and $\theta$ are derived via MATLAB for the particular worm parameters, these are substituted into the equation of worm helicoid shape $\Sigma_{1}$, and the instantaneous contact line can be drawn using the visual function of MATLAB. The instantaneous contact lines of worm helicoid $\Sigma_{1}$ and worm wheel tooth surface $\Sigma_{2}$ are depicted in Fig. 5.

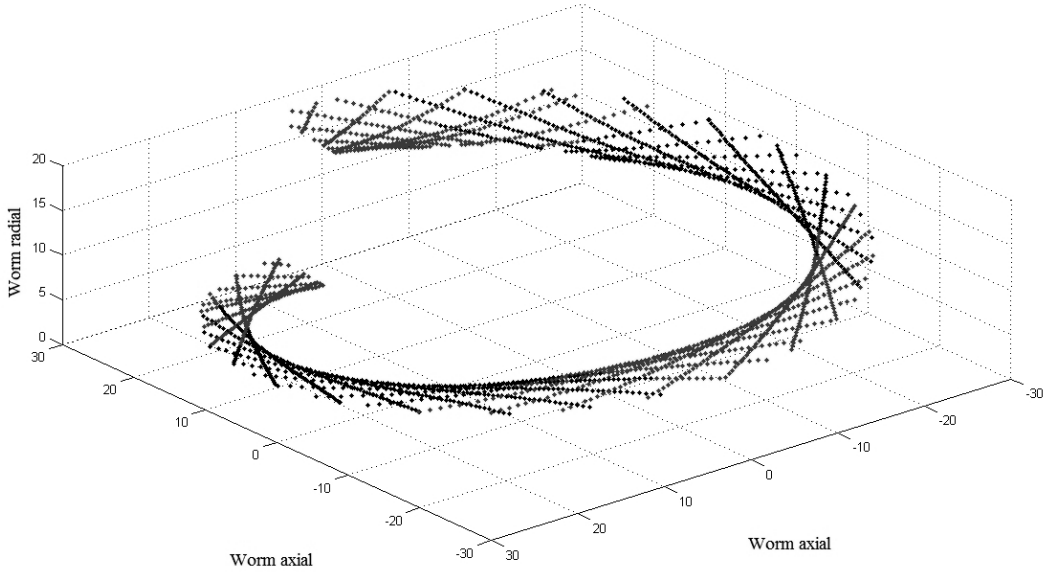

a

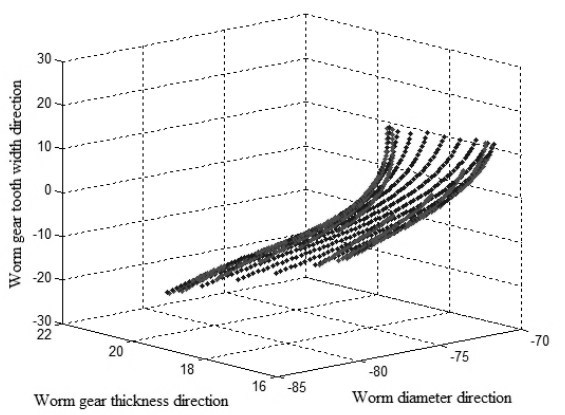

b

Fig. 5. Instantaneous contact lines of worm helicoid $\Sigma_{1}$ (a) and worm wheel tooth surface $\Sigma_{2}$ (b). 


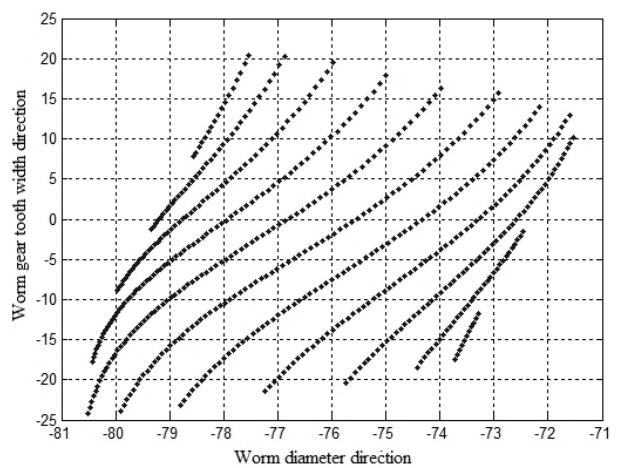

Fig. 6. Projection of the instantaneous contact line onto $\sigma_{2}\left(O_{2}, \vec{i}_{2}, \vec{k}_{2}\right)$.

The projection of instantaneous contact line of surface $\Sigma_{2}$ onto the plane coordinate system $\sigma_{2}\left(O_{2}, \vec{i}_{2}, \vec{k}_{2}\right)$ is shown in Fig. 6. The instantaneous contact line is coherent, which means that the worm drive contact is continuous and adapted to the process of meshing from the tooth top to the root. It can be seen from the plotted instantaneous contact lines that the helix angle $\alpha$ has a strong effect on the instantaneous contact line distribution of surface $\Sigma_{2}$ : variation in the value of $\alpha$ results in the respective variation of the distribution of the instantaneous contact line on $\Sigma_{2}$. If the value of $\alpha$ is varied with other conditions being unchanged, the distributions of the instantaneous contact line on $\Sigma_{2}$ can be obtained for $\alpha=3^{\circ}$ and $\alpha=20^{\circ}$, as is shown in Fig. 7. As compared with Fig. 6 , the meshing area for $\alpha=3^{\circ}$ is smaller and the instantaneous contact line variation is intensive after the decrease in $\alpha$, which results in the deteriorated meshing performance. At $\alpha=20^{\circ}$, the meshing area is increased, the instantaneous contact line is longer, and its distribution gets more uniform with the helix angle $\alpha$, improving the local meshing performance.

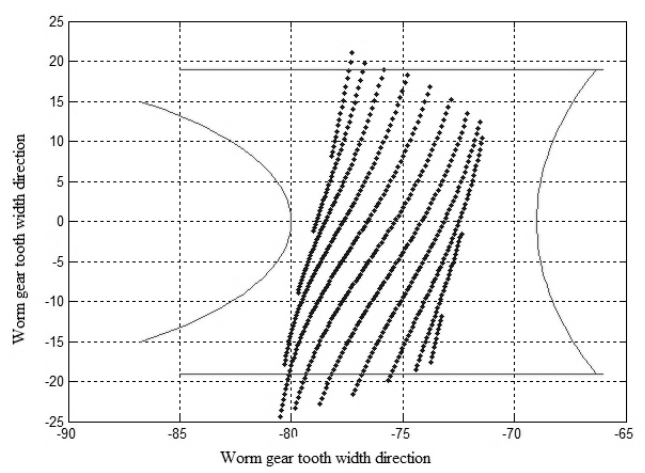

a

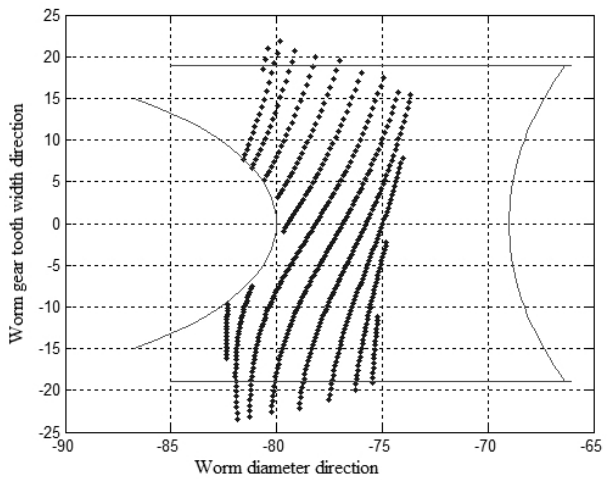

b

Fig. 7. Distribution of instantaneous contact lines for $\alpha=3^{\circ}$ (a) and $\alpha=20^{\circ}$ (b).

3. Numerical Analysis of Partial Meshing Quality Parameters of Worm Gear. After obtaining the instantaneous contact lines of the worm wheel with a meshing process, five meshing points are selected from each instantaneous contact line, as shown in Fig. 8.

The instantaneous contact line can be described as follows:

$$
K_{12 N}=\frac{N_{\alpha 1}^{2}+N_{\alpha 2}^{2}}{\psi},
$$




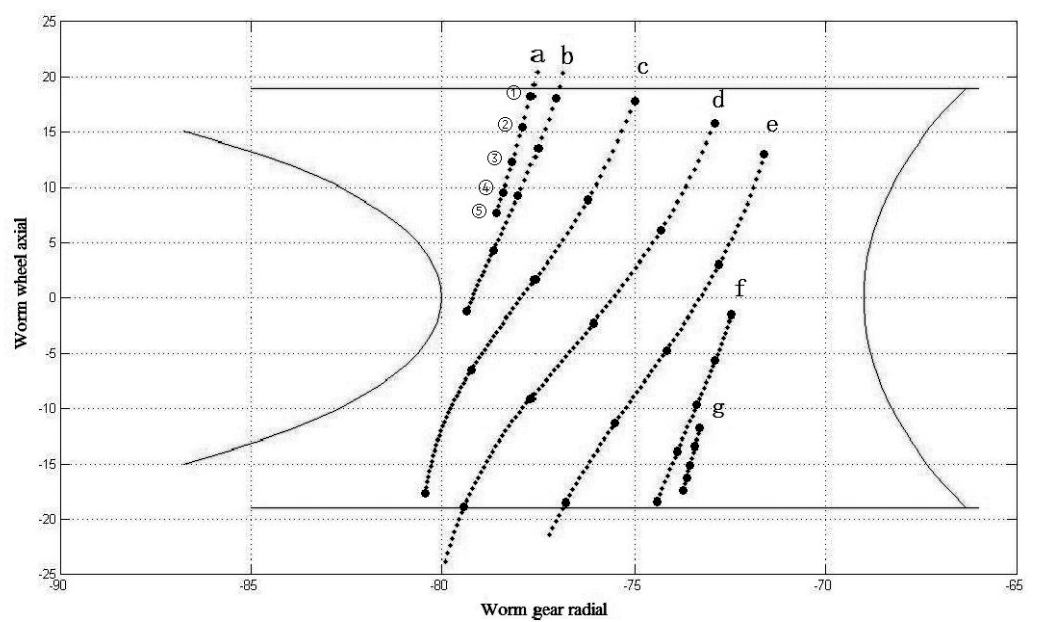

Fig. 8. Worm wheel instantaneous contact line.

where

$$
\begin{gathered}
N_{\alpha 1}=\left(K_{\alpha 1} \alpha_{x 1}+\tau_{\alpha 1} \alpha_{x 2}\right) v_{12 x}+\left(K_{\alpha 1} \alpha_{y 1}+\tau_{\alpha 1} \alpha_{y 2}\right) v_{12 y}+ \\
+\left(K_{\alpha 1} \alpha_{z 1}+\tau_{\alpha 1} \alpha_{z 2}\right) v_{12 z}+\frac{\alpha_{y 2}}{i_{12}}+\alpha_{z 2}, \\
N_{\alpha 2}=\left(\tau_{\alpha 1} \alpha_{x 1}+K_{\alpha 2} \alpha_{x 2}\right) v_{12 x}+\left(\tau_{\alpha 1} \alpha_{y 1}+K_{\alpha 2} \alpha_{y 2}\right) v_{12 y}+ \\
+\left(\tau_{\alpha 1} \alpha_{z 1}+K_{\alpha 2} \alpha_{z 2}\right) v_{12 z}-\frac{\alpha_{y 2}}{i_{12}}-\alpha_{z 2}, \\
\psi=\psi\left(u, \theta, \varphi_{1}\right) .
\end{gathered}
$$

The sliding angle $\theta_{v t}$ is derived as

$$
\theta_{v t}=\arcsin \left(-\frac{v_{12 x} N_{x}+v_{12 y} N_{y}+v_{12 z} N_{z}}{\sqrt{N_{\alpha 1}^{2}+N_{\alpha 2}^{2}} \sqrt{v_{12 x}^{2}+v_{12 y}^{2}+v_{12 z}^{2}}}\right) .
$$

The induced curvature and slip angle of the five points on each instantaneous contact line are calculated as in [11]. The results obtained are shown in Table 2 and imply that the calculated values of the induced curvature on the contact line are positive, indicating the availability of this part of the contact line on the tooth surface of the worm wheel. By comparing the induced curvature values, it can be seen that the curvature value of the contact line decreases with the angle $\varphi_{1}$, which indicates that the contact stress decreases gradually when the worm wheel enters the meshing state. The contact stress value at the straight end of the worm gear is lower than that of the end of the joint. The sliding angle increases with the angle $\varphi_{1}$, which can improve the lubrication conditions. The lubrication effect is more pronounced at the gear end of the worm wheel, as compared to homing end.

The sliding angle value can also influence the lubrication conditions of the worm. In Table 2, the maximum and minimum slip angles are $76^{\circ}$ and $9.84^{\circ}$, respectively, which strongly indicate good lubrication conditions for the major parts of the straight worm drive. 
Y. He, G. F. Li, Y. P. Zhao, et al.

$\mathrm{T}$ a b 1 e 2

Values of $K_{12 N}$ and $\theta_{v t}$ for the Worm Gear under Study

\begin{tabular}{|c|c|c|c|c|c|c|c|c||}
\hline \hline \multirow{2}{*}{ Point } & \multirow{2}{*}{ Parameter } & \multicolumn{7}{|c||}{ Angle } \\
\cline { 3 - 9 } & & $a$ & $b$ & $c$ & $d$ & $e$ & $f$ & $g$ \\
\cline { 3 - 9 } & & 3.6 & 3.8 & 4.2 & 4.6 & 5 & 5.4 & 5.6 \\
\hline \multirow{2}{*}{$(1)$} & $K_{12 N}$ & 0.0099 & 0.0061 & 0.0018 & 0.0005 & 0.0014 & 0.0088 & 0.0347 \\
\cline { 2 - 9 } & $\theta_{v t}, \operatorname{deg}$ & 43.27 & 39.95 & 22.81 & 39.88 & 76.59 & 69.25 & 68.80 \\
\hline \multirow{2}{*}{$(2)$} & $K_{12 N}$ & 0.0159 & 0.0176 & 0.0193 & 0.0041 & 0.0046 & 0.0193 & 0.02 \\
\cline { 2 - 9 } & $\theta_{v t}, \operatorname{deg}$ & 41.32 & 35.34 & 13.13 & 40.17 & 74.71 & 69.92 & 69.20 \\
\hline \multirow{2}{*}{$(3)$} & $K_{12 N}$ & 0.0304 & 0.06 & 0.0208 & 0.0073 & 0.146 & 0.0568 & 0.0115 \\
\cline { 2 - 9 } & $\theta_{v t}, \operatorname{deg}$ & 39.03 & 31.79 & 9.84 & 36.27 & 73.41 & 71.05 & 69.86 \\
\hline \multirow{2}{*}{$(4)$} & $K_{12 N}$ & 0.0778 & 0.086 & 0.0085 & 0.0021 & 0.0036 & 0.0087 & 0.0076 \\
\cline { 2 - 9 } & $\theta_{v t}, \operatorname{deg}$ & 36.64 & 27.65 & 12.63 & 26.85 & 71.14 & 72.61 & 70.58 \\
\hline \multirow{2}{*}{$(5)$} & $K_{12 N}$ & 0.2697 & 0.0516 & 0.0035 & 0.0007 & 0.0011 & 0.0037 & 0.006 \\
\cline { 2 - 9 } & $\theta_{v t}, \operatorname{deg}$ & 35.44 & 26.98 & 20.22 & 10.76 & 65.76 & 74.51 & 71.09 \\
\hline \hline
\end{tabular}

It is possible to reduce the induced curvature and slip angle by increasing the helix parameter $p$ of the worm normal straight part. This will somewhat increase the spiral angle $\alpha$ range, but reduce the curvature. Meanwhile, the reduction of the guide cylinder radius $r_{0}$ can greatly increase the value of the slip angle and decrease the value of the induced curvature, as is shown in Table 3.

$\mathrm{T}$ a $\mathrm{b} 1$ e 3

Values of $A$ and $B$ for the Worm Pair under Study

\begin{tabular}{|c|c|c|c|c|c|c||}
\hline Variable & \multirow{2}{*}{ Parameter } & \multicolumn{5}{|c|}{ Point } \\
\cline { 3 - 7 } & & (1) & (2) & (3) & (4) & (5) \\
\hline \multirow{2}{*}{$p=2$} & $K_{12 N}$ & 0.0014 & 0.0046 & 0.1461 & 0.0036 & 0.0011 \\
\cline { 2 - 7 } & $\theta_{v t}, \operatorname{deg}$ & 76.59 & 74.71 & 73.41 & 71.14 & 65.76 \\
\cline { 2 - 7 } & $K_{12 N}$ & 0.0012 & 0.0039 & 0.0479 & 0.0027 & 0.0008 \\
\hline \multirow{2}{*}{$\alpha=20^{\circ}$} & $\theta_{v t}, \operatorname{deg}$ & 79.29 & 78.69 & 77.88 & 75.78 & 69.93 \\
\cline { 2 - 7 } & $K_{12 N}$ & 0.001 & 0.0026 & 0.035 & 0.0021 & 0.0005 \\
\cline { 2 - 7 }$r_{0}=1.25$ & $\theta_{v t}, \operatorname{deg}$ & 79.54 & 78.81 & 78.09 & 75.92 & 70.35 \\
\cline { 2 - 7 } & $K_{12 N}$ & 0.0009 & 0.0024 & 0.0033 & 0.0018 & 0.0006 \\
\cline { 2 - 7 } & $\theta_{v t}, \operatorname{deg}$ & 79.64 & 78.95 & 78.19 & 76.65 & 70.27 \\
\hline
\end{tabular}

Conclusions. The results obtained made it possible to draw the following conclusions.

1. The instantaneous contact line of the straight worm pair is continuous, which confirms its feasibility, uniform contact stress distribution, and good lubrication conditions.

2. The helix angle value has a strong effect on the instantaneous contact line distribution of worm wheel tooth surface. Its variation changes the respective variation of the instantaneous contact line distribution, which allows one to adjust worm gear parameters for light, medium, and heavy-duty operation conditions.

3. An increase in the helix angle results in the larger meshing area and length of the instantaneous contact line. The latter distribution will be more uniform with an increase in the helix angle, which improves the local meshing performance. 
4. Further studies on the worm performance influencing factors are envisaged within the framework of the presented MATLAB-based numerical simulation approach.

Acknowledgments. The research work in this paper was fully supported by the National Natural Science Foundation of China under Grant No. 51475083.

1. L. V. Mohan and M. S. Shunmugam, "Geometrical aspects of double enveloping worm gear drive," Mech. Mach. Theory, 44, No. 11, 2053-2065 (2009).

2. Y. P. Zhao and Z. Zhao, "Computer aided analysis on the meshing behavior of a height-modified dual-torus double-enveloping toroidal worm drive," Comput. Aided Design, 42, No. 12, 1232-1240 (2010).

3. V. I. Medvedev, A. E. Volkov, M. A. Volosova, and O. E. Zubelevich, "Mathematical model and algorithm for contact stress analysis of gears with multi-pair contact," Mech. Mach. Theory, 86, 156-171 (2015).

4. W. K. Jehng, "Computer solid modeling technologies applied to develop and form mathematical parametric tooth profiles of bevel gear and skew gear sets," J. Mater. Process. Tech., 122, Nos. 2-3, 160-172 (2002).

5. Y. P. Zhao, J. Y. Kong, G. F. Li, et al., "Computerized simulation of tooth contact and error sensitivity investigation for ease-off hourglass worm drives," Comput. Aided Design, 44, No. 8, 778-790 (2012).

6. J. Brauer, "Transmission error in anti-backlash conical involute gear transmissions: a global-local FE approach," Finite Elem. Anal. Des., 41, No. 5, 431-457 (2005).

7. S. Strzelecki and Z. Towarek, "The effect of shaft unbalance on the operation of worm gear rolling bearings," Int. J. COMADEM, 13, No. 3, 2-9 (2010).

8. Y. P. Zhao, J. Y. Kong, G. F. Li, and T. C. Wu, "Tooth flank modification theory of dual-torus double-enveloping hourglass worm drives," Comput. Aided Design, 43, No. 12, 1535-1544 (2011).

9. G. F. Li, J. Liu, G. Z. Jiang, and H. H. Liu, "Numerical simulation of temperature field and thermal stress field in the new type of ladle with the nanometer adiabatic material," Adv. Mech. Eng., 7, No. 4, 1-13 (2015).

10. A. V. Stolyarov, "Differential geometry of distributions," J. Math. Sci., 207, No. 4, 635-657 (2015).

11. T. Lewiner, J. D. Gomes, Jr., H. Lopes, et al., "Curvature and torsion estimators based on parametric curve fitting," Comp. Graph., 29, No. 5, 641-655 (2005).

12. S. M. Vereshchaka, A. V. Deineka, and V. V. Danil'tsev, "Stress state of sealing ring of composite material with the specified physical and mechanical characteristics," Strength Mater., 48, No. 3, 371-374 (2016).

13. G. F. Li, P. X. Qu, J. Y. Kong, et al., "Influence of working lining parameters on temperature and stress field of ladle," Appl. Math. Inform. Sci., 7, No. 2, 439-448 (2013).

14. Y. P. Zhao, D. Z. Su, and Z. Zhang, "Meshing analysis and technological parameters selection of dual tori double-enveloping toroidal worm drive," Mech. Mach. Theory, 45, No. 9, 1269-1285 (2010).

15. D. Skrochocki and J. Tomczak, "Numerical simulation of rotary compression process of hollow balls," Strength Mater., 48, No. 4, 583-591 (2016). 